\title{
Spectrally efficient multicarrier modulation system for visible light communication
}

\author{
T. Deepa ${ }^{1}$, Harshita Mathur ${ }^{2}$, N.Bharathiraja ${ }^{3}$, K.A.Sunitha ${ }^{4}$ \\ ${ }^{1,4}$ SRM Institute of Science and Technology, India \\ ${ }^{2}$ SRMS College of Engineering Technology and Research, India \\ ${ }^{3}$ ARM College of Engineering and Technology, India
}

\begin{tabular}{l} 
Article Info \\
\hline Article history: \\
Received May 8, 2018 \\
Revised Dec 10, 2018 \\
Accepted Dec 26, 2018 \\
\hline
\end{tabular}

\section{Keywords:}

Delta-sigma modulator O-OFDM

Trellis coded modulation VLC

\begin{abstract}
Visible Light Communication (VLC) has become an accolade to its radio frequency counterpart. In VLC system, orthogonal frequency division multiplexing (OFDM) has drawn much attention, because of simple equalization, high spectral efficiency, high data rate and robustness to intersymbol interference (ISI). Besides, there are emerging applications that ought to be gotten with low latency and high reliability. To diminish power requirements with no transmission capacity extension, Trellis coded modulation (TCM) is utilized as a part of the framework in which the free distance of trellis diagram is equivalent to the minimum distance between the points of constellation focuses in partitioned subsets, which augments the coding gain up i.e. the performance parameter viably. TCM together with VLC-OFDM enhances the transmission execution in reasonable frameworks. In this paper, we propose OFDM which is based on TCM and is planned and exeuted for digitized OFDM frameworks by presenting delta sigma modulation (DSM) considering VLC channel. Simulation results show that the proposed TCM based VLC-OFDM offers incredible robustness against noises and nonlinear degradation.
\end{abstract}

Copyright $@ 2019$ Institute of Advanced Engineering and Science. All rights reserved.

\section{Corresponding Author:}

T. Deepa,

Department of Telecommunication Engineering,

SRM Institute of Science \& Technology, Chennai, India.

Email: deepa.t@ktr.srmuniv.ac.in

\section{INTRODUCTION}

Recent research and development focus of the access networks is being placed on the next generation passive optical networks based orthogonal frequency division multiplexing (PON-OFDM). It offers the best granularity so data can be apportioned to the clients by allotting mutually an arrangement of subcarriers in the frequency domain and in the time domain with various time slots [1]. Moreover, because of the adaptability in subcarrier-wise manipulation, it can likewise effortlessly empower the convergence among heterogeneous technologies (e.g. WiMAX, LTE etc.). In wireless frameworks, OFDM modulation has been adopted in wireless LAN (IEEE 802.11a/g, or also called Wi-Fi), WAN (IEEE 802.16e, or otherwise called WiMAX), and radio/video frameworks (DAB/DVB) received in many parts of the world [2], [3]. Generally, forwarding error correction (FEC) method has been utilized to enhance OFDM transmission system performance. Nonetheless, the codes in FEC, as a rule, present the overhead over the transmission rate. This implies the transmitted bandwidth must be reached out to fill in the coded system. Besides, the code with overhead will additionally enhance the signal, while the signal bandwidth will end up plainly more extensive. Thus, the spectrum efficiency will decrease. One extremely effective strategy for diminishing power requirements without expanding bandwidth is trellis coded modulation (TCM) [4], which permits exceptionally proficient and dependable digital transmission. In particular, TCM utilizes $M$ constellation 
points to transmit at a rate of $\log _{2}(M / 2)$ bits/symbol. Thus, a coding gain is acquired with no forfeit in bandwidth.

Visible light communication (VLC) frameworks utilize light emitting diode (LED) for enlightenment and information transmission in the meantime. From inquiring about investigations it is seen that it is utilized for the most part in indoors. The fundamental objective of optical OFDM (O-OFDM) is to build the data rate as per built-up optical modulation procedures. Visible light (VL) is the data communication medium which covers 400 and $800 \mathrm{THz}(750-375 \mathrm{~nm})$ in the electromagnetic range [5]. The optical transmission displays a capable option because of higher speed, better security, low power utilization, bigger data transfer capacity, and enhanced limit among others[6],[7].

In this paper, a TCM based delta sigma modulated OFDM system for VLC is proposed. Delta sigma modulation (DSM) based optical OFDM is designed and analyzed which offers astounding robustness against noises and nonlinear distortions. The digital signal is adaptable with the framework change not at all like the analog signal and is adding more security and encourages encryption on transmission. The proposed approach is to include a DSM in the transmission area in the TCM-OFDM VLC framework. Digitized TCMOFDM for machine to machine and car to car applications utilizing VL is a stage for the intention of evacuating inter-symbol interference (ISI) which is a type of signal mutilation in which one symbol meddles with back to back image symbol making the correspondence less dependable. The DSM is a strategy for changing over the continuous-time domain TCM-OFDM motion into digital signal. In this work, the execution of digitized TCM-OFDM framework is examined in terms of performance metrices such as peak to average power ratio (PAPR), bit error rate (BER), error vector magnitude (EVM), power spectral density (PSD) and signal to quantization noise ratio (SQNR).

The organization of the paper is as follows: Section 2 depicts the principles of TCM and DSM for OFDM systems. In Section 3, we describe the proposed system model for VLC channel utilizing the TCM performance model for optical OFDM. The VLC model is depicted in Section 4. Computer simulation results are provided in Section 5 and conclusion is presented in Section 6.

\section{PRINCIPLES OF MODULATIONS: TCM AND DSM}

For the conventional affirmation of TCM, the coding gain is proficient by using convolution encoder for increasing the constellation points of the modulation with coded input bits [8]. The coding gain can be generally written as,

$$
\gamma=\left[\frac{E_{\text {uncoded }}}{d_{\text {min }, \text { uncoded }}^{2}}\right] /\left[\frac{E_{\text {coded }}}{D_{f, \text { coded }}^{2}}\right]
$$

where,

$\mathrm{E}$ is the normalized average received energy,

$\mathrm{d}^{2}$ min represents the minimum distance between all pairs of two sequences,

$D_{f, c o d e d}^{2}$ is the minimum Euclidean distance between paths that diverge from any state and remerge at the same state in the trellis code.

Figure 1 exhibits the convolutional encoder of TCM-M ary modulation. TCM uses set partitionaing and small number of states at the transmitter and Viterbi decoding algorithm is applied to the trellis, with surviving partial paths corresponding to partial signal sequences that are closest to the received sequences.

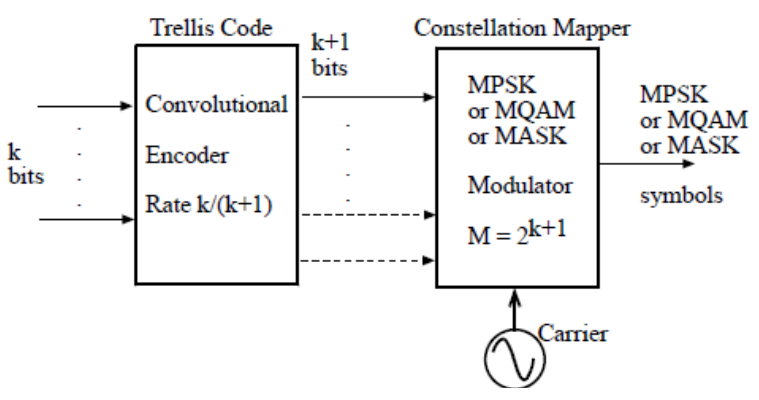

Figure 1. Convolutional encoder of TCM-M ary modulation 
DSM is a modulation technique for encoding analog signal into digital signal [9]-[12]. It is used to convert high bit-count, low-frequency digital signals into lower bit-count, higher-frequency digital signals as part of the process to convert digital signals into analog as part of a digital-to-analog converter (DAC). The DSM structure is depicted in Figure 2.

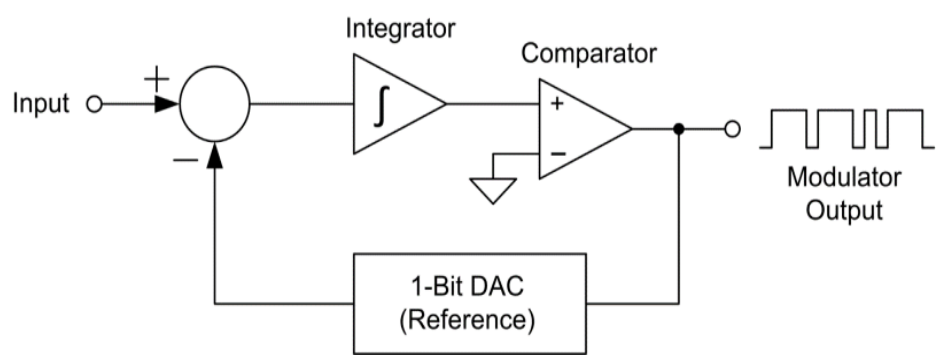

Figure 2. Delta Sigma Modulator

\section{PROPOSED TCM BASED VLC-OFDM}

The proposed block diagram of the TCM based VLC-OFDM is illustrated in Figure 3. In this segment, DSM is acquainted with engaging the transmission of a digitized TCM-OFDM signal by means of VLC channel. The DSM-analog to a digital converter (DSM-ADC) incorporates an interpolator, delta-sigma modulator, digital filter, and decimation. The analog TCM-OFDM signal is upsampled by an interpolation factor, $I_{\mathrm{f}}$. The upsampled signal is encoded into a digital signal by utilizing DSM and down sampled by decimation. The TCM-OFDM signal is recuperated by applying low pass filter (LPF) at the recipient. VLC as contrasted and RF advancements, is protected, does not constitute or experience the ill effects of electromagnetic interference, and offers an immense unregulated data transfer capacity between $400 \mathrm{THz}$ $(750 \mathrm{~nm})$ and $800 \mathrm{THz}(375 \mathrm{~nm})$.

For indoor VLC frameworks, dispersion with ISI impact can be displayed as baseband linear impulse response. This multipath contortion impact is generallyconcerned particularly for non-directional and non-LOS channel models. Indoor VLC channels are for the most part accepted as quasi-static. The VLC channel model is depicted in Figure 4.

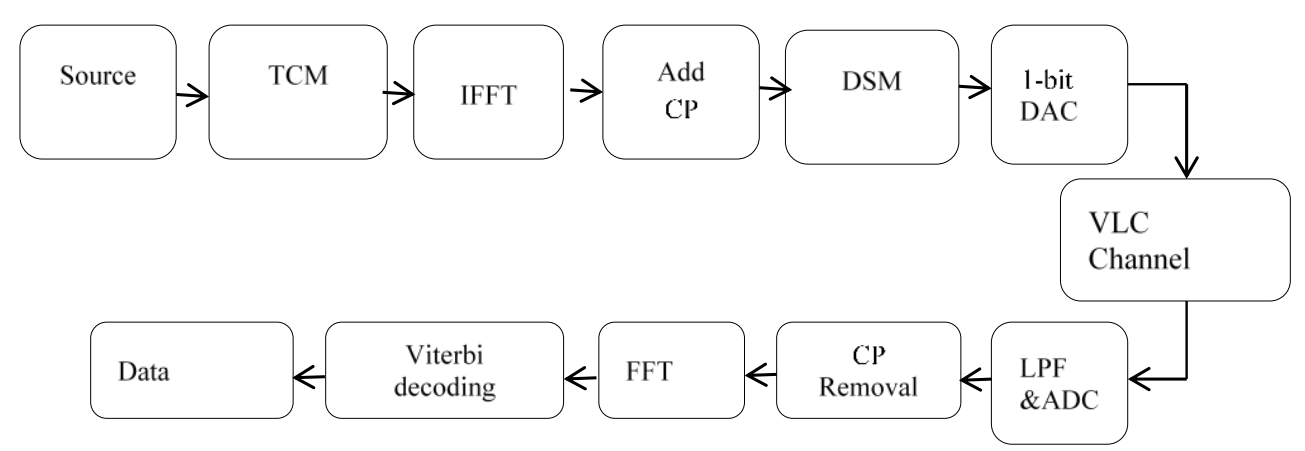

Figure 3. Proposed TCM-OFDM based DSM for VLC system

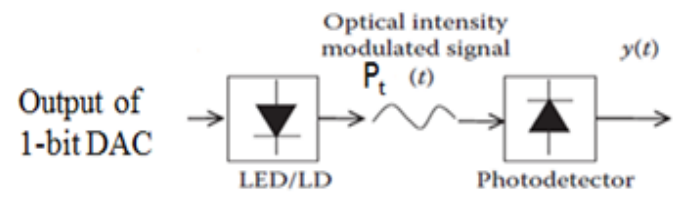

Figure 4. VLC channel model 


\section{VLC CHANNEL MODEL}

The received TCM based VLC-OFDM signal is given by [13],

$$
y(t)=R \cdot x(t) \otimes h(t)+n(t)
$$

where,

$\mathrm{R}$ is the photodetector'sresponsivity

$\mathrm{n}(\mathrm{t})$ is the Gaussian modeled noise which has ambient and preamplifier receiver noises

$\otimes$ is the convolution operation

$x(t)$ is the instantaneous input power $(x(t)>0)$

Given an emitter $\mathrm{E}$ and a recipient $\mathrm{R}$ in a situation free of reflectors as shown in Figure 5 with a huge separation $\mathrm{d}_{0, \mathrm{R}}$ between both, the LOS impulse response is written as[14]-[16],

$$
h^{o}(t ; E, R, \lambda)=\frac{1}{d_{(0, R)^{2}}} R_{E}(\varphi, m, \lambda) A_{e f f}(\Psi) \delta\left(t-\frac{d_{(0, R)}}{c}\right)
$$

where

$R_{E}(\varphi, m, \lambda)$ is the generalized Lambertian model utilized to approximate the radiation pattern of the emitter,

$\mathrm{c}$ is the speed of light,

$A_{e f f}(\Psi)$ is the successful signal accumulation area of the recipient, which is represented by,

$\mathrm{m}$ is the mode number of the radiation projection, which determines the directionality of the emitter.

The parameters used in the simulation framework for VLC is mentioned in the Table 1.

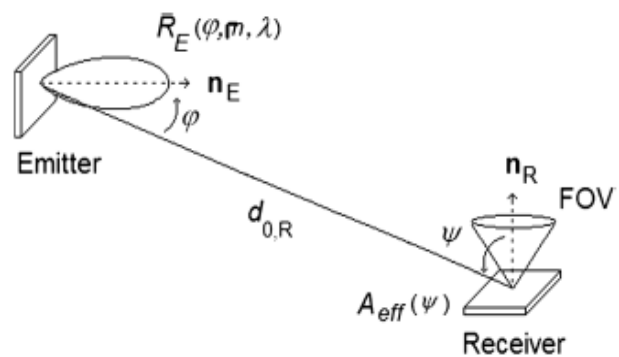

Figure 5. Geometry of emitter and receiver

Table 1. Simulation Parameters

\begin{tabular}{ll}
\hline \multicolumn{1}{c}{ VLC parameters } & \multicolumn{1}{c}{ Values } \\
\hline Input sequence & 10000 bits \\
Modulation & TCM -OFDM \\
IFFT/FFT size & 256 \\
Cyclic Prefix $(\mathrm{CP})$ size & 16 \\
Bipolar to unipolar conversion & DSM \\
Channel & VLC , AWGN \\
Oversampling Ratio (OSR) & 16 \\
Interpolation factor and decimation & 64 \\
Room Size $\left(\mathrm{L}^{*} \mathrm{~W}^{*} \mathrm{H}\right)$ & $5 \mathrm{~m} * 5 \mathrm{~m} * 3 \mathrm{~m}$ \\
Distance between emitter and receiver(m) & 1.5 \\
Transmitter Semi-angle at half power, $\varphi_{1}$, (radian $)^{*}$ & $\left(7.5^{*} \mathrm{pi}\right) / 180$ \\
Angle of incidence, $\psi$ & $\left(15^{*} \mathrm{pi}\right) / 180$ \\
FOV (field of view) of detector in half $($ Radian $)$ & $\left(20^{*} \mathrm{pi}\right) / 180$ \\
Power emitted by LED (mW) $\mathrm{P}_{\mathrm{E}}$ & 0.1 \\
Photodiode responsivity $(\mathrm{A} / \mathrm{W})$ & 0.55 \\
Physical area of the receiver, $\mathrm{A}_{\mathrm{R}}\left(\mathrm{m}^{2}\right)$ & $10^{-4} \mathrm{~m}^{2}$ \\
\hline
\end{tabular}




\section{RESULTS AND DISCUSSION}

The continuous time domain representation of OFDM signal after the input data is passed through OFDM transmitter is shown in Figure 6. It is observed that the digital signal when compared with the analog signal is more stable amplitude wise and is more likely to provide better data rates. Figure 7 shows comparison of the PSDs of digital VLC-OFDM with and without TCM which is an average power distributed as a function of frequency. It is observed that the SQNR performance for digitized TCM based OFDM for VLC system is better than system without TCM.

Figure 8 illustrates the BER performance of proposed system as the function of SNR. It is observed that the coding gain $(\mathrm{CG})$ of $3.5 \mathrm{~dB}$ is achieved in the proposed system. Figure 9(a) shows the CCDF of VLC-OFDM symbols with and without TCM. It is clearly shown that the VLC-OFDM with TCM achieves 10.6dB PAPR and system without TCM achieves 16dB PAPR at $10^{-1}$ CCDF level. Figure 9(b) andIllustrates the comparison of DSM based VLC-OFDM system performance with and without TCM. It is observed that VLC-OFDM system with TCM has lower PAPR value than the system without TCM. Also the analysis is done by taking into consideration the parameters like MERand EVM, which again shows the better performance of DSM based VLC-OFDM system with TCM.

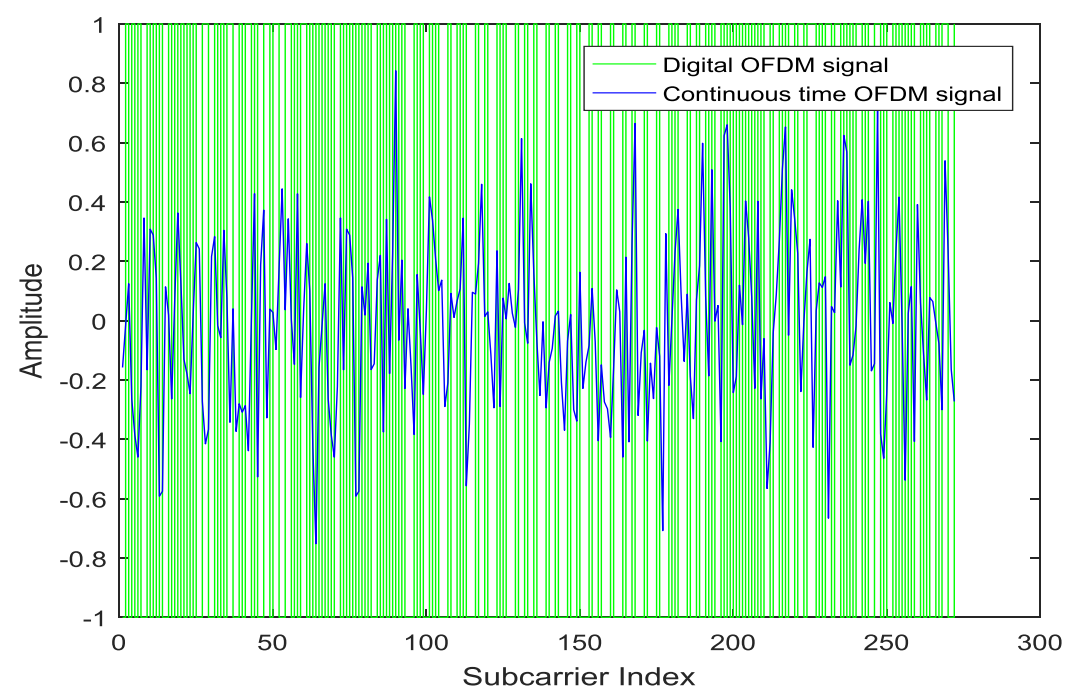

Figure 6. Representation of continuous time and digitized OFDM signal
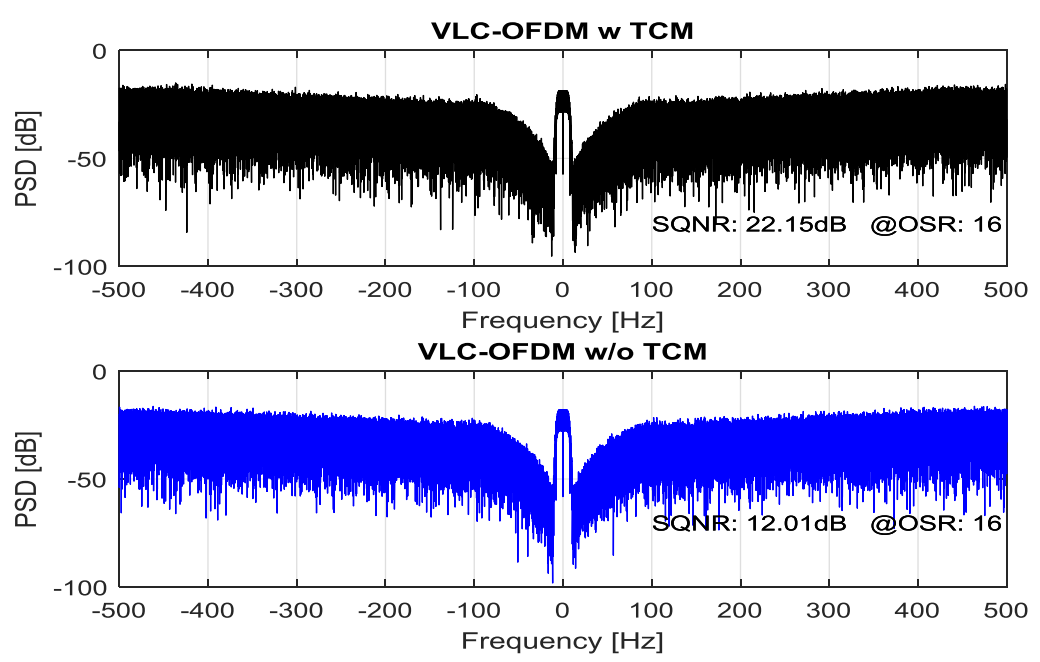

Figure 7. Comparison of PSDs of digital VLC-OFDM with and without TCM 


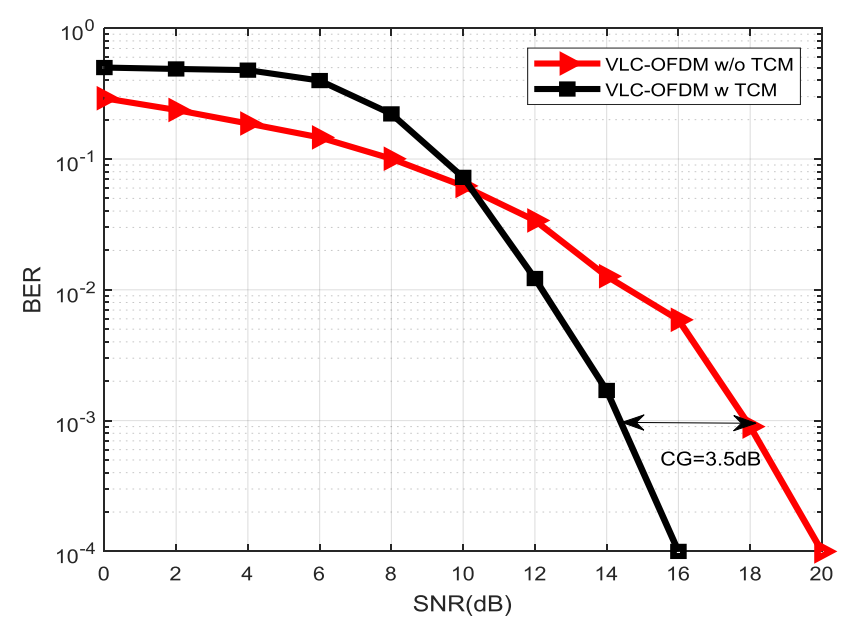

Figure 8. BER performance of VLC-OFDM with and without TCM

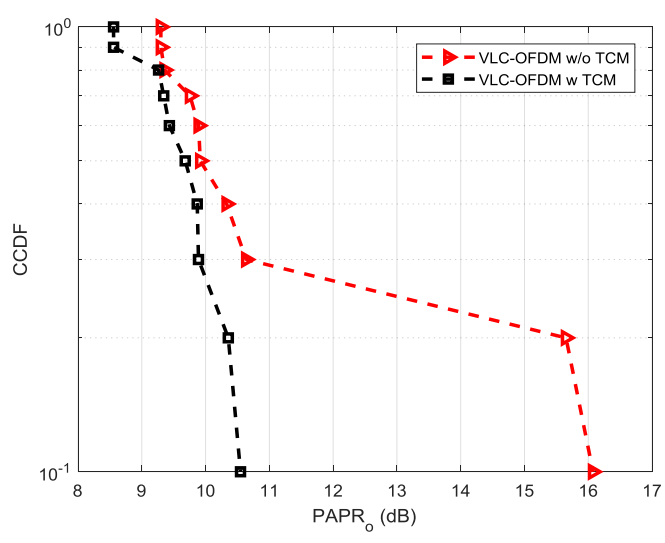

(a)
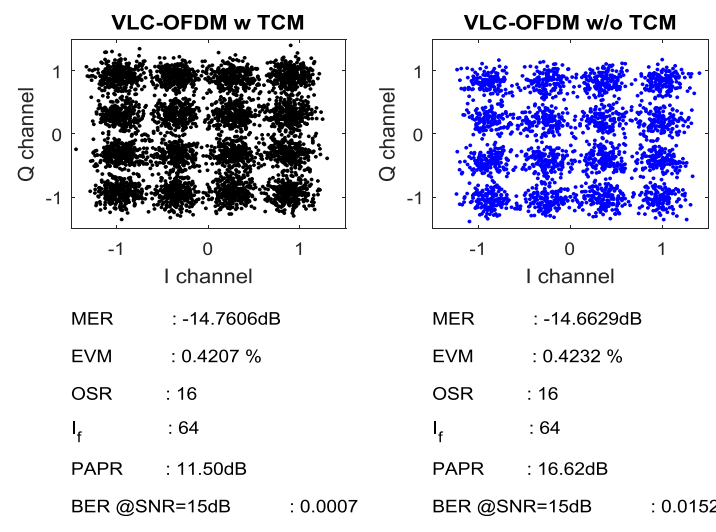

(b)

Figure 9. (a) CCDF of VLC-OFDM with and without TCM, (b) Received constellation diagram and metrices for DSM based VLC-OFDM with and without TCM

\section{CONCLUSION}

In this paper, a digital TCM-OFDM based on DSM channel has been proposed for VLC. The performance of digital OFDM system has been analyzed with and without TCM by means of simulation. Simulattion result has shown that the PAPR reduction has been achieved by $5.12 \mathrm{~dB}$ in the TCM based VLCOFDM system. It has also been observed that the BER performance has been improved in the case of VLCOFDM with TCM than without TCM system and $3.5 \mathrm{~dB}$ of coding gain has been achieved in the proposed system.

\section{REFERENCES}

[1] Z. Yu, Y. Lou, M. Chen, H. Chen, S. Yang and S. Xie, "Adaptive Three-Dimensional Optimization for Optical Direct-Detection OFDM," in Journal of Lightwave Technology, vol. 35, no. 9, pp. 1506-1512, May1, 12017.

[2] R Nee, R Prasad, “OFDM for Wireless Multimedia Communications”, 2000.

[3] Hocine Ait-Saadi, Jean-Yves Chouinard, AbderrazakGuessoum, "A PAPR Reduction for OFDM Signals Based on Self-Adaptive Multipopulation DE algorithm," International Journal of Electrical and Computer Engineering (IJECE), Vol. 7, No. 5, pp. 2651 - 2660, October 2017.

[4] Yiming Lou, Zhenming Yu, Minghua Chen, Hongwei Chen, Sigang Yang and ShizhongXie, "Experimental demonstration of 10-Gb/s direct detection optical OFDM transmission with Trellis-coded 8PSK subcarrier modulation," 2016 21st OptoElectronics and Communications Conference (OECC) held jointly with 2016 International Conference on Photonics in Switching (PS), Niigata, pp 1-3, 2016. 
ArsyadRamadhanDarlis, "Bidirectional Underwater Visible Light Communication," International Journal of Electrical and Computer Engineering (IJECE), Vol 8, No 6: December 2018.

[5] Sung Wan Koo, J. S. Park and Jin Young Kim, "High Speed VLC System Based on Turbo-Coded OFDM Scheme," 11th International Conference on Advanced Communication Technology, Phoenix Park, pp. 1534-1537, 2009.

[6] SuseelaVappangi, Venkata Mani Vakamulla, "Channel Estimation In ACO-OFDM Employing Different Transforms for VLC," AEU International Journal of Electronics and Communications, Volume 84, Pages 111-122, 2018. https://doi.org/10.1016/j.aeue.2017.11.016.

[7] X. Liu, Q. Yang, S. Chandrasekhar, and W. Shieh, "Transmission of 44-Gb/s Coherent Optical OFDM Signal with Trellis-Coded 32-QAM Subcarrier Modulation," in Proc. Opt. FiberCommun., 2010, Paper OMR3.

[8] HarshitaMathur, T Deepa, "OFDM-Based on Trellis-Coded Modulation for Optical Wireless Communication," International Conference on Intelligent Computing and Applications, pp-79-86, September 2018.

[9] Nasser ErfaniMajd, Hassan Ghafoorifard, Abbas Mohammadi, "Bandwidth Enhancement in Delta Sigma Modulator Transmitter Using Low Complexity Time-Interleaved Parallel Delta Sigma Modulator", $A E U$ International Journal of Electronics and Communications, Volume 69, Issue 7, Pages 1032-1038, 2015. https://doi.org/10.1016/j.aeue.2015.04.001.

[10] Jang,S G. Jo, J. Jung, B. Park and S. Hong, "A Digitized IF-Over-Fiber Transmission Based on Low- Pass DeltaSigma Modulation," IEEE Photonics Technology Letters, vol. 26, no. 24, pp. 2484-2487, Dec.15, 152014.

[11] T. Deepa, T. R. Rao, HarshitaMathur, P. Srivstava and B. T. Abe, "Performance Analysis of Digitized Multicarrier System for Optical Wireless Communication," 2017 Global Wireless Summit (GWS), Cape Town, South Africa, pp. 29-33, 2017. DOI: 10.1109/GWS.2017.8300293.

[12] Li R. Hu, H.B. Li, M. Luo Q. Yang and S.H. Yu, "Digital OFDM-PON Based on Delta-sigma Modulation Employing Binary IM-DD Channels Citation Information," IEEE Photonics Journal, 2016. DOI 10.1109/JPHOT.2016.2639040

[13] S. Alreesh, C. Schmidt-Langhorst, R. Emmerich, P. WilkeBerenguer, C. Schubert and J. K. Fischer, "Four-Dimensional Trellis Coded Modulation for Flexible Optical Communications," Journal of Lightwave Technology, vol. 35, no. 2, pp. 152-158, Jan.15, 152017.

[14] L. Wei, H. Zhang and B. Yu, "Optimal Bit-and-power Allocation Algorithm for VLC-OFDM System," Electronics Letters, vol. 52, no. 12, pp. 1036-1037, 692016.

[15] Ram Sharma, A. Charan Kumari, Mona Aggarwal, Swaran Ahuja, "Optimal LED Deployment for Mobile Indoor Visible Light Communication System: Performance Analysis," AEU - International Journal of Electronics and Communications, Volume 83, Pages 427-432, 2018. 